\title{
Análise das Recomendações de Manuais de Aleitamento Infantil: Possibilidades e Desafios
}

\author{
Veronica Aparecida Pereira ${ }^{1}$ \\ Faculdade de Ciências Humanas da Universidade Federal da Grande Dourados, \\ Dourados, MS, Brasil \\ Olga Maria Piazentin Rolim Rodrigues \\ Departamento de Psicologia da Universidade Estadual Paulista Júlio de Mesquita Filho, \\ Bauru, SP, Brasil \\ Millena Lima Donato \\ Fabiana Calixtro Maruchi \\ Priscilla Joyce Vieira do Amaral \\ Universidade Federal da Grande Dourados, Dourados, MS, Brasil
}

\begin{abstract}
Resumo
Apesar do incentivo da Organização Mundial da Saúde para garantir a amamentação, há contraindicações que podem torná-la temporária ou inviável. Considerando-se os esforços dos órgãos públicos brasileiros para atendimento desta meta, buscou-se no presente artigo: (a) analisar em documentos oficiais do Ministério da Saúde e secretarias estaduais do Brasil, informações importantes sobre o aleitamento natural e artificial; (b) identificar em que medida os documentos disponíveis apresentam informações a mães que amamentam e mães que oferecem aleitamento artificial. Selecionou-se onze manuais nacionais e oito estaduais, publicados de 2007 a 2013, a partir dos endereços eletrônicos das secretarias de saúde, nacionais e estaduais do Brasil. A maioria dos estados referendou os documentos já disponíveis no Ministério da Saúde. Em todos houve grande enfoque quanto aos benefícios da amamentação exclusiva até o sexto mês de vida. Outras informações atenderam também ao critério de alimentação complementar, atendimento à nutriz por profissionais da saúde, divulgação de programas em prol da amamentação, direitos das mães e características culturais e étnicas. As informações sobre aleitamento artificiais limitam-se ao relato de contraindicações para o mesmo.
\end{abstract}

Palavras-chave: Contraindicações da amamentação, amamentação, aleitamento artificial.

\section{Analysis of Recommendations from Breastfeeding Handbooks: Possibilities and Challenges}

\begin{abstract}
Despite the encouragement of the World Health Organization to ensure breastfeeding, there are contraindications that may make it temporarily or unfeasible. Considering the efforts of Brazilian public agencies to meet this goal, we sought to in this paper intends to (a) analyze official documents of the Ministry of Health and State Departments in Brazil, relevant information on natural and artificial

Endereço para correspondência: Rua Portugal, 445, Dourados, MS, Brasil 79826-370. Fone: (67) 2310-2310. E-mail: veronica.ufgd.tci@gmail.com, olgarolim@uol.com.br, mi_donatto@hotmail.com, fabimaruchi@ hotmail.com e pri-joyce@hotmail.com
\end{abstract}


feeding; (b) identify in what measure available documents present information to breastfeeding mothers and to mothers that provide artificial feeding. Eleven national manuals and eight state was selected, published from 2007 to 2013, from the online addresses of Health Departments at the national and State levels in Brazil. In all of there was a great emphasis on the benefits of exclusive breastfeeding until the sixth month of life. Other pieces of information refer to the criterion of complementary feeding, care for the mother provided by healthcare professionals, dissemination of pro breastfeeding programs, rights of the mothers and cultural and ethnic features. Information on artificial feeding is limited to report contraindications to it.

Keywords: Contraindications for breastfeeding, breastfeeding, artificial feeding.

\section{Análisis de las Recomendaciones de Manuales de Lactancia Infantil: Posibilidades y Desafios}

\section{Resumen}

A pesar del estímulo de la Organización Mundial de la Salud para garantizar la lactancia materna, hay contraindicaciones que pueden hacer que sea de forma temporal o inviable. Teniendo en cuenta los esfuerzos de los organismos públicos brasileños para cumplir con este objetivo, hemos tratado en este artículo: (a) analizar documentos del Ministerio de Sanidad y de secretarias estaduales de Brasil, informaciones sobre lactancia materna natural y artificial; (b) identificar en que medida los documentos presentan informaciones a madres lactantes y madres que ofrecen lactancia artificial. Fueron seleccionados once manuales nacionales y ocho estaduales, en el periodo de 2007 a 2013, a partir de las direcciones electrónicas de las secretarias de sanidad, nacionales y estaduales de Brasil. La mayoría de les Estados refrendó los documentos del Ministerio de Sanidad. En los manuales nacionales y estaduales fueron destacados los beneficios de la lactancia materna exclusiva hasta el sexto mes de vida. Otras informaciones se ocuparon del criterio de alimentación complementaria y de los cuidados a la madre por los profesionales sanitarios, divulgación de programas a favor de la lactancia, derechos de la madre y características culturales y étnicas. Las informaciones sobre lactancia artificial se limitan al relato de sus contraindicaciones.

Palabras clave: Contraindicaciones de lactancia materna, lactancia materna, lactancia artificial.

$\mathrm{Na}$ atualidade, o debate sobre a importância da amamentação é crescente, tanto do ponto de vista cultural (Almeida \& Novak, 2004) como nutricional (Corona \& Conde, 2013). A Organização Mundial da Saúde - OMS (World Health Organization [WHO]) orienta que a amamentação seja a alimentação exclusiva do bebê, até os seis meses de idade. Após esse período, recomenda a introdução de alimentação complementar devendo a amamentação, sempre que possível, ser mantida até os dois anos de idade. Esta providência visa, principalmente, diminuir os altos índices mundiais de desnutrição infantil, aumento da imunidade e diminuição da mortalidade infantil (WHO, 2009). Para tanto, Almeida e Novak (2004) alertaram que os fatores biológicos precisam estar contextualizados à realidade da mulher-mãe-nutriz e a relação estabelecida com seu bebê, de modo a favorecer a adaptação à condição materna e novas exigências interpostas.

Santiago (2013) apresentou dados preocupantes sobre a duração média do aleitamento materno no Brasil. Segundo um estudo realizado em 2008, a média da duração do aleitamento materno exclusivo no Brasil é de apenas 54,11 dias, sendo que, a prevalência de mães que permanecem amamentando até os quatro ou seis meses é de 23,3 e $9 \%$, respectivamente. Isto foi observado mesmo após um longo período de divulgação 
na mídia sobre a importância da amamentação, visando a conscientização da população. A compreensão destes índices, segundo o autor, pode estar associada a falta de preparo da rede de saúde na orientação às mães, problemas de saúde da mãe e o afastamento do trabalho inferior às necessidades do bebê.

Em contrapartida, estudiosos indicam outras preocupações em países com plena adesão ao aleitamento materno exclusivo, como na Índia. Thakur, Holambe e Wadagale (2015), em estudo realizado com uma população de 197 mães indianas, com média de 24,17 anos de idade, encontraram amamentação exclusiva até os cinco meses de idade do bebê. A preocupação, por sua vez, esteve relacionada ao índice de $53,63 \%$ das mães que não haviam introduzido alimentos complementares à alimentação do bebê, após os seis meses, o que seria importante para o seu desenvolvimento. Neste caso, a preocupação se depara com o grande risco de desnutrição e vulnerabilidade dos bebês pela falta de nutrientes indispensáveis ao seu crescimento e indisponíveis no aleitamento materno exclusivo nesta idade.

As metas estabelecidas pela OMS (WHO, 2009), sobre a manutenção da amamentação exclusiva até o sexto mês de vida do bebê, requerem ações, no âmbito de políticas públicas que garantam não só a amamentação como o atendimento das necessidades nutricionais do bebê após este período.

No que se refere ao despreparo dos profissionais da área da saúde, a OMS apontou a falta de habilidade para orientar devidamente a puérpera frente às dificuldades do início da amamentação. Eles podem não saber como ajudar a mãe a iniciar e manter o aleitamento materno exclusivo, recomendando introdução precoce de complementos ou, ainda, de forma aberta ou velada, promover substitutos do leite materno (WHO, 2009). Nos apontamentos de Almeida e Novak (2004), tais condutas levariam a importação da cultura do desmame precoce e fortalecimento de mitos acerca da amamentação.

Em uma experiência positiva da formação de profissionais frente a esta demanda, Davanzo, Pierpaolo e Travan (2014) descreveram um estudo realizado na Itália. Os autores ressaltaram a importância da formação de profissionais de saúde na orientação e manejo das questões relativas ao início da amamentação. Para tanto, estabeleceram protocolos de registro de necessidades das mães no período de pós-parto e práticas da equipe técnica que possam favorecer o início e manutenção da amamentação. Entre as preocupações identificadas destacaram: cuidados essenciais à mãe no período do pós-parto, no qual deve-se favorecer o contato entre mãe e bebê pelo toque, contato 'pele a pele'; atenção e orientação durante o período em que o bebê apresenta perda de peso e a mãe pode ter receio de não ter leite suficiente; orientações caso a mãe tenha necessidade de ingestão de medicamentos que possam apresentar efeitos colaterais para o bebê e, problemas relacionados à saúde do bebê, como má-absorção e alergias. Para as questões apresentadas, a equipe técnica, ainda no período de internação da mãe, deverá estar suficientemente preparada para orientá-la sobre possíveis períodos de interrupção provisória do aleitamento, oferta de leite humano pelo banco de leite ou fórmulas especializadas e a manutenção da produção e ordenha do leite materno para oferta futura ao bebê.

A partir das indicações de Davanzo et al. (2014), cumpre destacar que a orientação poderá estender-se no período de alta, indicando programas de atenção às mães durante o início da amamentação. Considerando-se o período necessário para descida do leite, que em alguns casos pode chegar até 72 horas (Fundo das Nações Unidas para a Infância [Unicef], 2008), é comum que muitas mães saiam da maternidade sem terem iniciado efetivamente a amamentação, enfrentando sozinhas uma série de dificuldades quanto à ordenha, formato do bico, dores, rachaduras no mamilo entre outras.

A orientação às mães se faz necessária uma vez que muitas vezes as mulheres não se encontram devidamente preparadas e esclarecidas sobre a amamentação. Rahalkar, Phalke e Phalke (2014) referem que os benefícios da amamentação dependerão de fatores como o início, duração e idade em que ocorrerá o desmame, sendo importante compreender que fa- 
tores culturais e econômicos estão diretamente ligados a estas práticas. Ao conduzirem um estudo com 150 nutrizes, na Índia, durante dois meses, identificaram mediante aplicação de questionário que as práticas mais adequadas de amamentação estavam relacionadas ao melhor nível de formação das mães.

A grande ênfase no aleitamento materno se baseia em inúmeros estudos que o consideram como um alimento ideal principalmente por suas vantagens nutricionais e condições de imunidade. O Ministério de Saúde do Brasil e a Organização Pan-Americana da Saúde (Opas) reafirmaram os indicativos da OMS quanto a importância da amamentação exclusiva até o sexto de mês de vida do bebê (Ministério da Saúde, 2002; Opas, 2013). Além das vantagens biológicas e nutricionais, os estudos destacaram a contribuição para o desenvolvimento global e saudável do bebê (Cotrim, Venancio, \& Escuder, 2002; Opas, 2013). Destacaram também sua influência no desenvolvimento psicomotor, cognitivo e afetivo da criança (Araujo, Del Fiaco, Pimentel, \& Schmitz, 2004; Bayardo, Peixoto, \& Corrêa, 2003; Carrascoza, Costa, \& Moraes, 2005; Opas, 2013).

Além dos fatores supramencionados advindos da amamentação, estão também presentes os fatores culturais, os quais atribuem à mãe as obrigações de doação completa à maternidade. Neste âmbito, a impossibilidade de amamentar pode ser interpretada por algumas mulheres como se elas tivessem uma dívida de ordem social e biológica com seu filho. De certa forma, a mulher sente essa cobrança, mesmo que de forma implícita, por profissionais e familiares (Bispo, Bispo, \& Pepe, 2010).

A impossibilidade efetiva de amamentar decorre, principalmente, devido aos altos índices de infecção apresentados pela mãe, a qual seria transmitida pelo leite materno. Nesta condição, a OMS estabeleceu algumas medidas para prevenção da transmissão de doenças indicando a substituição da amamentação pelo uso de fórmulas apropriadas até o segundo ano de vida para crianças nascidas de mães portadoras de doenças como AIDS, Hepatite B e outras doenças passíveis de contaminação através do leite humano (Unicef, 2008). Porém, nem sempre essas fórmulas são acessíveis, devido ao seu alto custo. O que se percebe, muitas vezes, é a incidência de aleitamento artificial indevido, com utilização de leite impróprio para o consumo de lactentes. $\mathrm{O}$ uso de leite impróprio encontra-se associado à desnutrição, alto índice de alergias, adoecimento e mortalidade infantil (Diaz, Patrício, \& Fagundes-Neto, 2002; Santiago, 2013).

Embora não seja uma interdição a amamentação, o retorno da mãe ao trabalho muitas vezes encontra-se associado ao desmame precoce. Segundo Brasileiro, Ambrosano, Marba e Possobon (2012), o índice de mães-nutrizes trabalhadoras que oferecem aleitamento materno exclusivo é inferior a 10\%. No Brasil, em 1988, a legislação apresentou avanços significativos assegurando à mulher a licença maternidade de 120 dias (Senado Federal, 1988). Em 2008, a Lei federal $\mathrm{n}^{\circ} 11.770$ buscou incentivar as empresas a concederem a licença maternidade por seis meses, período que atenderia a primeira exigência da OMS. Somada a essa iniciativa, orientou-se que as empresas viabilizassem vale-creche às mães-nutrizes, com intervalos de trinta minutos para amamentação durante o turno de trabalho. Contudo, as condições de deslocamento e acesso às creches ainda não se mostram suficientes para manutenção efetiva da amamentação depois dos seis meses (Santiago, 2013). Na amostra investigada por Brasileiro et al. (2012), a maioria das mães efetuou o desmame antes dos quatro meses, preparando-se para o retorno ao trabalho.

Embora a mudança na legislação brasileira ainda não atenda plenamente as exigências da OMS, as medidas iniciais apontam para diminuição do quadro de adoecimento e mortalidade infantil, os quais mostravam-se elevados na década de 80. Segundo Batista e Silva (2007) as iniciativas do Ministério da Saúde baseadas na defesa do aleitamento materno foram responsáveis pela criação do Programa Nacional de Incentivo ao Aleitamento, valorizando e difundindo essa prática pela cultura brasileira (Opas, 2013).

Sabendo-se da importância do aleitamento materno e da impossibilidade de algumas mães em realizar esta prática, é possível que mães impossibilitadas de amamentar possam sentir- 
-se culpadas por não serem capazes de prover o alimento de seu filho e as condições necessárias ao seu bom desenvolvimento. Gestantes e puérperas, que não podem amamentar, afirmam que essa restrição lhes acarreta culpa, frustrações, sofrimentos, desejos interrompidos, impotência e sonhos desfeitos (Paiva \& Galvão, 2004). A interdição à amamentação pode trazer várias repercuções psicológicas à mãe, sendo necessário promover conscientização sobre os comportamentos presentes durante o aleitamento que sejam favoráveis a estruturação de vínculo mãe-bebê, independente da forma de aleitamento. Nesta tarefa, a atuação dos profissionais da saúde será de extrema importância, desvendando e enfrentando mitos e medos da mãe sobre esta interdição.

Em busca de apontar subsídios às mães impossibilitadas de amamentar, o presente artigo buscou: (a) realizar uma análise das informações disponíveis em manuais sobre aleitamento para bebês, no Brasil, no período de 2007 a 2013, de modo a identificar informações sobre a qualidade do leite, quantidade, condições de higiene, posição do bebê e ganhos psicológicos na alimentação natural e artificial e, (b) comparar os resultados e indicar informações pertinentes às mães impossibilitadas de amamentar.

\section{Método}

Para realização da revisão bibliográfica foram selecionados manuais de aleitamento disponíveis nos sítios das Secretarias Nacionais e Estaduais da Saúde no Brasil, no período de 2007 a 2013.

Foram selecionados onze manuais nacionais e oito estaduais. O critério de seleção para os nacionais foi a lacuna temporal. Nas secretarias estaduais, percebeu-se que a maioria referendava os manuais nacionais. Assim sendo, aquelas que indicaram manuais próprios na temática amamentação (cartilha ou manual), dentro do período em estudo, foram selecionadas. No estado do Paraná foi selecionado um manual do município de Curitiba pelo fato deste ser recomendado pela secretaria estadual de saúde.
A análise de dados foi organizada de duas formas. Inicialmente, foram pré-estabelecidas categorias de análise para o conteúdo exposto acerca do aleitamento materno e artificial, sendo estas: (a) qualidade do leite; (b) quantidade a ser oferecida ao bebê; (c) condições de higiene; (d) frequência; (e) posição do bebê; (f) introdução de complementos alimentares e, $(\mathrm{g})$ ganhos psicológicos. As categorias foram quantificadas e descritas em relação ao seu conteúdo. Na segunda análise, buscou-se identificar os principais conteúdos oferecidos pelos manuais que extrapolavam as categorias em estudo. Em ambas as análises realizou-se uma descrição quantitativa e qualitativa.

\section{Resultados e Discussão}

Após a leitura dos manuais nacionais e regionais, foi quantificada a ocorrência das categorias previamente selecionadas no presente estudo. Como observado na Tabela 1, a primeira categoria de análise refere-se à qualidade do leite. Todos os manuais nacionais ressaltaram a qualidade do leite materno em suas propriedades nutritivas, diminuição do risco de adoecimento e adequação às necessidades do bebê (Ministério da Saúde, 2007, 2009, 2010a, 2010b, 2010c, 2011a, 2011b, 2012a, 2012b, 2013a, 2013b; Unicef, 2008). Embora o manual da Unicef (2008) seja internacional, foi veiculado a partir de um portal nacional, sendo o mesmo considerado para a análise. Quanto ao leite artificial, alguns manuais apontaram as fórmulas, quando o aleitamento for contraindicado para o bebê (Ministério da Saúde, 2009, 2010c, 2013b). A qualidade das fórmulas nos manuais mostra-se inferior ao leite materno no quesito imunidade e disponibilidade, sendo este último também destacado nos manuais estaduais (Secretaria Estadual de Saúde de Minas Gerais, 2012; Secretaria Estadual de Saúde do Mato Grosso do Sul, 2007a, 2007b, 2007c, 2007d). Alertou-se, também, quanto ao uso de campanhas indevidas em estabelecimentos comerciais, as quais poderiam incentivar o uso de fórmulas independente da condição da mãe e do bebê (Ministério da Saúde, 2013b). 
Tabela 1

Análise dos Manuais Informativos sobre Aleitamento Infantil

\begin{tabular}{|c|c|c|c|c|c|c|}
\hline \multirow{2}{*}{ Categorias } & \multicolumn{3}{|c|}{$\begin{array}{l}\text { Manuais Nacionais } \\
\text { (11) }\end{array}$} & \multicolumn{2}{|c|}{$\begin{array}{c}\text { Manuais Estaduais } \\
\text { (8) }\end{array}$} & \multirow[b]{2}{*}{$\mathrm{AM}$} \\
\hline & Tipo de leite & Nacional & PR & MG & MS & \\
\hline \multirow{2}{*}{ Qualidade do leite } & Materno & $11 / 11$ & $1 / 1$ & $1 / 1$ & $4 / 4$ & $1 / 1$ \\
\hline & Artificial & $3 / 11$ & $0 / 1$ & $0 / 1$ & $0 / 4$ & $0 / 1$ \\
\hline \multirow{2}{*}{ Quantidade de leite } & Materno & $3 / 11$ & $1 / 1$ & $1 / 1$ & $2 / 4$ & $0 / 1$ \\
\hline & Artificial & $1 / 11$ & $0 / 1$ & $0 / 1$ & $0 / 4$ & $0 / 1$ \\
\hline \multirow{2}{*}{$\begin{array}{l}\text { Frequência de } \\
\text { amamentação }\end{array}$} & Materno & $5 / 11$ & $1 / 1$ & $1 / 1$ & $4 / 4$ & $0 / 1$ \\
\hline & Artificial & $1 / 11$ & $0 / 1$ & $0 / 1$ & $0 / 4$ & $0 / 1$ \\
\hline \multirow{2}{*}{ Posição do bebê } & Materno & $5 / 11$ & $1 / 1$ & $1 / 1$ & $1 / 4$ & $0 / 1$ \\
\hline & Artificial & $2 / 11$ & $0 / 1$ & $0 / 1$ & $0 / 4$ & $0 / 1$ \\
\hline \multirow{2}{*}{$\begin{array}{l}\text { Higienização (mama/ } \\
\text { objetos) }\end{array}$} & Materno & $3 / 11$ & $1 / 1$ & $1 / 1$ & $0 / 4$ & $0 / 1$ \\
\hline & Artificial & $3 / 11$ & $0 / 1$ & $1 / 1$ & $0 / 4$ & $0 / 1$ \\
\hline \multirow{2}{*}{ Complemento } & Materno & $5 / 11$ & 0 & $1 / 1$ & $3 / 4$ & $0 / 1$ \\
\hline & Artificial & $3 / 11$ & 0 & 0 & $3 / 4$ & $0 / 1$ \\
\hline \multirow{2}{*}{ Ganhos psicológicos } & Materno & $1 / 11$ & 0 & $1 / 1$ & $2 / 4$ & $0 / 1$ \\
\hline & Artificial & $1 / 11$ & 0 & $0 / 0$ & $0 / 4$ & $0 / 1$ \\
\hline
\end{tabular}

Nota. PR - Paraná, MG - Minas Gerais, MS - Mato Grosso do Sul e AM - Amazonas.

Quanto à quantidade de leite oferecida ao bebê, observou-se que a minoria (7/18) dos manuais nacionais abordou esta categoria relacionada ao leite materno. As indicações sobre a quantidade não são precisas, requerendo da mãe a habilidade de reconhecer quando seu filho tem fome. Para tanto, o manual Unicef (2008) descreve a condição do recém-nascido o qual, inicialmente, terá seu ciclo de sono interrompido pela fome. A mãe, por sua vez, deverá atentar-se para que o intervalo de sono não exceda três horas no primeiro mês de vida. O mesmo manual indica que o tempo necessário para que um bebê esvazie uma mama é de quatro minutos, o que é alterado pelo tempo em que ele efetivamente suga o leite e o tempo em que ele permanece com a mama como se fosse uma 'chupeta'. Há o indicativo de que regime de oferta do aleitamento materno deverá ser livre, com horários flexíveis, atendendo a necessidade do bebê (Ministério da Saúde, 2009; Unicef, 2008). Isso poderá acarretar diariamente várias mamadas, sendo necessário que a mãe esteja descansada e totalmente liberada para este ato. $\mathrm{O}$ regime de oferta em demanda livre é referendado por $50 \%$ dos manuais estaduais (Secretaria Estadual de Saúde do Mato Grosso do Sul, 2007a, 2007b; Secretaria Estadual de Saúde de Minas Gerais, 2012; Secretaria Municipal da Saúde de Curitiba - PR, 2011). Sobre a quantidade a ser oferecida em regime de aleitamento artificial, o tema foi pouco abordado, apareceu em apenas um dos manuais nacionais (Ministério da Saúde, 2013b). Esta citação aparece na sequência das restrições à amamentação. Em outros manuais, como da Unicef (2008), trabalhou-se mais as questões relativas à restrição temporária da amamentação, para a qual recomenda-se a oferta de leite humano pasteurizado por translactação. Esta, "consiste em um recipiente contendo leite (preferencialmente humano pasteurizado) colocado entre as mamas da mãe e conectado ao mamilo por meio de uma sonda" (Ministério da Saúde, 2009, p. 38). Outra indicação aponta 
para o uso de fórmulas oferecidas em copo ou colher, mantendo-se a estimulação da produção do leite materno a partir da ordenha (Unicef, 2008). Quando a restrição é definitiva, são citadas rapidamente o uso de fórmulas, contudo, sem indicação de quantidades específicas.

A frequência de oferta do aleitamento materno foi abordada pela maioria dos manuais, apontando, sobretudo para a oferta em demanda livre, respeitando-se o ritmo e necessidade de cada bebê (Ministério da Saúde, 2009, 2010a; Secretaria Estadual de Saúde de Minas Gerais, 2012; Secretaria Estadual de Saúde do Mato Grosso do Sul, 2007a, 2007b, 2007c, 2007d). Já a frequência do aleitamento artificial foi abordada por apenas um manual nacional (Ministério da Saúde, 2013b).

A posição do bebê foi abordada nos manuais nacionais e regionais. Quando aparece, durante a amamentação, encontra-se associada às explicações sobre a formação da mama, pega do bebê, posições de ventilação e respiração. Outras observações referem-se à necessidade de observação dos comportamentos do bebê durante o aleitamento de modo a assegurar-se de uma boa sucção (Ministério da Saúde, 2009, 2010a, 2012a). A posição do bebê na oferta de aleitamento artificial foi pouco citada e, quando ocorreu, esteve associada à preocupação de boa deglutição e prevenção de asfixia, refluxo e infecções do canal auditivo (Ministério da Saúde, 2009; Unicef, 2008). Em ambas as situações não foram abordados os aspectos subjetivos deste contato, como a possibilidade de comunicação não verbal entre a mãe e bebê, contato visual, toque e atenção que se fazem necessários. A posição do bebê atendendo os critérios de segurança também foi abordada nos manuais estaduais e no municipal avaliados (Secretaria Estadual de Saúde do Mato Grosso do Sul, 2007b; Secretaria Municipal da Saúde de Curitiba, 2011). Nesta última citação, refere-se ao fato da indígena poder estar sentada durante a amamentação, o que dispensa o esforço do preparo do leite artificial. Por isso, trata mais da posição da mãe do que do bebê propriamente. A ênfase se dá na liberdade da indígena em amamentar em qualquer circunstância, visto que o leite está sempre pronto e nas condições ideais para o bebê.

Os cuidados de higiene foram mais detalhadamente explicados no manual do Ministério da Saúde (2012a). Os demais manuais nacionais abordaram o tema ao discutir sobre o cuidado das mamas, principalmente durante as atividades de ordenha e armazenamento de leite materno para pasteurização. As práticas de higiene relacionada ao cuidado e oferta de fórmulas ou leite humano pasteurizado referiram-se principalmente aos cuidados de esterilização de copos, utensílios e mamadeiras (Ministério da Saúde, 2009, 2010a; Unicef, 2008). Entre os manuais estaduais, esta categoria foi abordada nos manuais de Minas Gerais e do Paraná (Secretaria Estadual de Saúde de Minas Gerais, 2012; Secretaria Municipal da Saúde de Curitiba, 2011). Nos manuais do estado do Mato Grosso do Sul, o uso de mamadeiras é apontado como uma das principais causas de infecção, diarreia e até mortalidade infantil, no entanto, não são descritas informações específicas sobre a higienização das mesmas (Secretaria Estadual de Saúde do Mato Grosso do Sul, 2007a, 2007b, 2007c).

A alimentação complementar foi abordada parcialmente nos manuais nacionais. Nos estaduais, foi destacada em Minas e no Mato Grosso do Sul. As citações relativas ao leite materno indicaram a introdução de alimentos após o sexto mês de aleitamento materno exclusivo como importante medida para nutrição e saúde infantil (Ministério da Saúde, 2009, 2010a, 2010b; Secretaria Estadual de Saúde de Minas Gerais, 2012; Secretaria Estadual de Saúde do Mato Grosso do Sul, 2007a, 2007b, 2007c), corroborando os estudos de Thakur et al. (2015) e as orientações de Opas (2013). Nos manuais de etnias indígenas (Secretaria Estadual de Saúde do Mato Grosso do Sul, 2007a, 2007b, 2007c) houve o cuidado em descrever o tipo de alimentação complementar relacionados aos produtos disponíveis na aldeia, valorizando produtos por ele produzidos e isentos de conservantes e agrotóxicos. O aleitamento artificial foi citado principalmente como alternativa às contraindicações da amamentação (Ministério da Saúde, 2010a, 2010c, 2013b). 
Em relação aos ganhos psicológicos da amamentação ou aleitamento artificial, verificou-se que este é o item menos abordado entre os manuais nacionais (Ministério da Saúde, 2009; Unicef, 2008). Os comportamentos presentes no ato de alimentação do bebê, na forma em que se apresentam, estão mais relacionados à sobrevivência e nutrição, sem que sejam cuidados os aspectos de vinculação entre mãe e filho, muito fortes durante esta atividade. Entre os manuais estaduais, os manuais de saúde indígena, os apontamentos da etnia Kaiwá ressaltaram a oferta de leite materno como um ato capaz também de transmitir amor e carinho (Secretaria Estadual de Saúde do Mato Grosso do Sul, 2007a). No manual dos Terenas, além da questão nutricional, imunidade e ênfase em alimentação complementar saudável com produtos naturais, também presentes nos demais manuais $(2007 \mathrm{a}, 2007 \mathrm{c})$ a amamentação é descrita como capaz de favorecer o "vinculo afetivo entre a mãe e o filho" (2007b, p. 14). A foto que segue a citação não apresenta a oferta do seio e sim outro alimento entregue à uma criança maior, sugerindo que este vínculo ultrapassa a condição do aleitamento. O manual do estado de Minas Gerais destaca que os bebês que são amamentados ao peito, além dos benefícios à saúde como um todo, apresentam "melhor desenvolvimento emocional, social, motor e da inteligência" (Secretaria Estadual de Saúde de Minas Gerais, 2012, p. 5).

$\mathrm{Na}$ segunda análise, outros aspectos foram destacados como relevantes nos manuais de aleitamento. O resultado desta análise, com estruturação de categorias após a leitura dos manuais, encontra-se dividido em duas partes: Tabela 2, análise dos manuais nacionais e Tabela 3 , análise dos manuais regionais.

Tabela 2

Principais Temas Abordados nos Manuais Nacionais sobre o Aleitamento

\begin{tabular}{lc}
\hline \multicolumn{1}{c}{ Temas } & Frequência \\
\hline Recomendações para evitar o uso de acessórios para aleitamento artificial & $2 / 11$ \\
Ordenha, higiene e armazenamento de leite materno. & $2 / 11$ \\
Problemas frequentes na amamentação / manejo / manutenção da amamentação & $2 / 11$ \\
Mitos e tabus sobre a amamentação & $2 / 11$ \\
Serviços de saúde - apoio à amamentação, cuidados à mãe que amamenta & $3 / 11$ \\
Industrialização de leites e fórmulas - normas para promoção e distribuição em estabelecimentos & $1 / 11$ \\
de saúde & $1 / 11$ \\
Tipos de aleitamento & $11 / 11$ \\
Importância do leite materno exclusivo até o sexto mês & $2 / 11$ \\
Retorno da mãe ao trabalho, direitos da mãe que amamenta & $1 / 11$ \\
Aleitamento em condições especiais: gêmeos, nova gravidez, prematuridade, deficiência, etc. \\
Restrições à amamentação & $3 / 11$ \\
Saúde do recém-nascido & $2 / 11$ \\
Introdução de alimentos após o sexto mês e orientações sobre o desmame & $3 / 11$ \\
\hline
\end{tabular}

Entre os temas destacados na Tabela 2 é possível identificar grande ênfase na importância do aleitamento materno exclusivo até o sexto mês, atendendo às recomendações da OMS (WHO, 2009) e Opas (2013). Em três deles, segue-se a importância de orientar às mães sobre o processo inicial de desmame, com a introdução de alimentos também importantes à saúde e nutrição do bebê, visto que após o sexto mês a manutenção exclusiva da amamentação seria insuficiente (Opas, 2013; Thakur et al., 2015) e também dificultada pelo retorno das mães ao tra- 
balho. Também estiveram presentes as contraindicações à amamentação e os serviços de apoio à mãe que amamenta, principalmente dos bancos de leite, salientando a importância da formação da equipe técnica no acompanhamento da mãe- -nutriz (Davanzo et al., 2014; Santiago, 2013). Alguns abordaram sobre os direitos da mãe que retorna ao trabalho e as demais temáticas visaram indicar cuidados necessários para a manutenção da amamentação.

Tabela 3

Principais Temas Abordados nos Manuais Regionais sobre o Aleitamento

\begin{tabular}{lc}
\hline \multicolumn{1}{c}{ Temas } & Frequência \\
\hline Nutrição, Saúde e Cuidados na Gravidez & $2 / 8$ \\
Características e cuidados com a Mama & $2 / 8$ \\
Importância e Vantagens da Amamentação & $8 / 8$ \\
Impacto hormonal & $2 / 8$ \\
Orientação para mães que voltarão a trabalhar/direitos das mães & $2 / 8$ \\
Ordenha & $4 / 8$ \\
Contraindicação ao aleitamento & $2 / 8$ \\
Desmame & $2 / 8$ \\
Programas de aleitamento & $1 / 8$ \\
Informações culturais específicas & $4 / 8$ \\
\hline
\end{tabular}

Além das orientações destacadas nos manuais nacionais, sobretudo em relação à importância da amamentação exclusiva, os manuais estaduais ressaltam algumas condições específicas sobre o período pré-natal, cuidados com a mama, saúde da mãe (cuidados com a mama, ordenha, impacto hormonal), retorno ao trabalho e contraindicações à amamentação (Tabela 3). O link da secretaria de saúde do Amazonas direcionou para o Plano Estadual de Saúde, no qual foi divulgado o Programa Amamenta Brasil como prioritário na atenção primária à saúde, atendendo às estratégias de promoção da saúde materna e infantil (Secretaria Estadual de Saúde do Amazonas, 2012). As informações culturais específicas referem-se ao cuidado na divulgação das informações sobre a amamentação respeitando a cultura indígena no tocante às etnias Kaiwá, Terena e Kadweu (Secretaria Estadual de Saúde do Mato Grosso do Sul, 2007a, 2007b, 2007c) bem como da cultura dos surdos, tornando acessíveis as informações através da Língua Brasileira de Sinais (Secretaria Estadual de Saúde do Mato Grosso do Sul, 2007d).

\section{Considerações Finais}

A análise dos manuais selecionados para análise no presente estudo apontou para uma diversidade de informações acerca da importância do aleitamento materno exclusivo até o sexto mês de vida do bebê e recomendações para alimentação complementar até os dois anos de idade, atendendo as recomendações da OMS e Opas (Opas, 2013; WHO, 2009).

Contudo, para os casos de contraindicações à amamentação, temporária ou permanente, as informações às mães que precisam recorrer ao aleitamento artificial parecem insuficientes. Sabendo-se que as reservas em banco de leite humano seriam insuficientes para atender a demanda de mães impossibilitadas de amamentar, informações precisas e seguras sobre o uso de fórmulas e de programas que possam oferecer o leite alternativo na rede pública são imprescindíveis. Além disso, faz-se necessário minimizar os possíveis sentimentos de culpa e fracasso da mãe que não amamenta, orientando-a sobre os comportamentos que estão presentes durante o 
aleitamento artificial e como estes irão favorecer o vínculo mãe-bebê. A descrição mais detalhada destes comportamentos, como o toque, contato visual, carinho e responsividade deveriam estar presentes em todos os manuais que tratam da amamentação.

Acariciar, beijar, tocar e olhar para o bebê com a finalidade de manter contato e demonstrar afeto da mãe para seu filho, pode fazer com que o aleitamento seja considerado um marco importante para a formação do vínculo mãe-bebê e para a manutenção de uma interação afetiva e saudável entre a díade. Quando ocorre de forma bem sucedida, a amamentação pode ser considerada fator facilitador da função materna de se vincular ao recém-nascido, atendendo suas necessidades fundamentais e possibilitando o desenvolvimento da capacidade deste para se relacionar com outros objetos de afeto (Locatelli \& Costa, 2008).

A mãe que oferece o leite artificial também precisa saber como fazê-lo, pois carinho, toque, contato visual e demonstração de afeto não estão agregados ao seio materno. Atender ao recém-nascido de forma responsiva, mesmo quando for necessário usar um instrumento para seu aleitamento (mamadeira, copinho ou colher) poderá ser efetivo se esse comportamento conseguir aproximá-lo de seu cuidador.

Espera-se que os achados do presente estudo possam evidenciar as informações de grande relevância para o aleitamento materno e incentivar propostas de campanhas de amamentação que também se atentam à realidade de mães que não podem amamentar.

\section{Referências}

Almeida, J. A. G., \& Novak, F. R. (2004). Amamentação: Um híbrido natureza-cultura. Jornal de Pediatria, 80(5), 119-125. doi:10.2223/JPED.1242

Araujo, M. F. M., Del Fiaco, A., Pimentel, L. S., \& Schmitz, B. A. S. (2004). Custo e economia da prática do aleitamento materno para a família. Revista Brasileira de Saúde Materno Infantil, 4(2), 135-141. doi:10.1590/S151938292004000200003
Batista, B. C., \& Silva, L. R. (2007). Sentimentos de mulheres soropositivas para HIV diante da impossibilidade de amamentar. Escola Anna Nery Revista de Enfermagem, 11(2), 268-275. doi:10.1590/s1414-81452007000200013

Bayardo, R. A., Peixoto, L. F. S., \& Corrêa, M. S. N. P. (2003). Aleitamento natural e artificial: Considerações gerais [Resumo]. JBC Jornal Brasileiro de Clínica Odontológica Integrada, 7(39), 257-290.

Bispo, T. M. S., Bispo, R. G., \& Pepe, A. L. (2010). Os aspectos psicológicos da interdição à amamentação. Psicologia. PT, O portal dos Psicólogos. Recuperado em http://www.psicologia.pt/ artigos/textos/TL0163.pdf

Brasileiro, A. A., Ambrosano, G. M. B., Marba, S. T. M., \& Possobon, R. F. (2012). A amamentação entre filhos de mulheres trabalhadoras. Revista de Saúde Pública, 46(4), 642-648. doi:10.1590/ S0034-89102012000400008

Carrascoza, K. C., Costa, A. L., Jr., \& Moraes, A. B. A. (2005). Fatores que influenciam o desmame precoce e a extensão do aleitamento materno. $E s$ tudos de Psicologia (Campinas), 22(4), 433-440. doi:10.1590/S0103-166X2005000400011

Corona, L. P., \& Conde, W. L. (2013). O efeito do aleitamento materno na composição corporal de menores de três anos em São Paulo, Brasil. Journal of Human Growth and Development, 23(3), 276-281.

Cotrim, L. C., Venancio, S. I., \& Escuder, M. M. L. (2002). Uso de chupeta e amamentação em crianças menores de quatro meses no estado de São Paulo. Revista Brasileira de Saúde Materno Infantil, 2(3), 245-252. doi:10.1590/S151938292002000300005

Davanzo, R., Pierpaolo, B., \& Travan, L. (2014). Integrating health care practices with the promotion of breastfeeding. Journal of Pediatric and Neonatal Individualized Medicine, 3(2), 1-5.

Diaz, N. J., Patricio, F. S., \& Fagundes-Neto, U. (2002). Colite alérgica: Características clínicas e morfológicas da mucosa retal em lactentes com enterorragia. Arquivos de Gastroenterologia, 39(4), 260-267. doi:10.1590/ S0004-28032002000400010

Fundo das Nações Unidas para a Infância. (2008). Manual de aleitamento materno. Recuperado em https://www.unicef.pt/docs/manual_aleitamento.pdf 
Locatelli, B. M. E. S., \& Costa, P. J. (2008). O processo de amamentação e suas implicações para a mãe e seu bebê. Mental, 10(6), 85-102.

Ministério da Saúde. (2002). Guia alimentar para crianças menores de 2 anos. Brasília, DF: Autor. Recuperado em http://bvsms.saude.gov. br/bvs/publicacoes/10_passos.pdf

Ministério da Saúde. (2007). Promovendo o aleitamento materno. Brasília, DF: Autor. Recuperado em http://www.redeblh.fiocruz.br/media/ albam.pdf

Ministério da Saúde. (2009). Saúde da criança: Nutrição infantil, aleitamento materno e alimentação complementar. Cadernos de Atenção Básica, 23. Recuperado em http://bvsms.saude.gov.br/ bvs/publicacoes/saude_crianca_nutricao_aleitamento_alimentacao.pdf

Ministério da Saúde. (2010a). Dez passos para uma alimentação saudável. Guia alimentar para crianças menores de dois anos. Brasília, DF: Autor. Recuperado em http://bvsms.saude.gov. br/bvs/publicacoes/10_passos.pdf

Ministério da Saúde. (2010b). Cartilha para a mãe trabalhadora que amamenta. Brasília, DF: Autor. Recuperado em http://bvsms.saude.gov. $\mathrm{br} / \mathrm{bvs} /$ publicacoes/cartilha_mae_trabalhadora amamenta.pdf

Ministério da Saúde. (2010c). Amamentação e uso de medicamentos e outras substâncias: Série A. Normas e Manuais Técnicos (2. ed.). Brasília, DF: Autor. Recuperado em http://bvsms.saude. gov.br/bvs/publicacoes/amamentacao_uso_ medicamentos_2ed.pdf

Ministério da Saúde. (2011a). Além da sobrevivência: Práticas integradas de atenção ao parto, benéficas para a nutrição e a saúde de mães e crianças. Brasília, DF: Autor. Recuperado em http://bvsms.saude.gov.br/bvs/publicacoes/ alem_sobrevivencia_atencao_parto.pdf

Ministério da Saúde. (2011b). Rede amamenta Brasil, os primeiros passos (2007-2010). Série I. História da Saúde. Brasília, DF: Autor. Recuperado em http://www.ibfan.org.br/legislacao/pdf/ doc-750.pdf

Ministério da Saúde. (2012a). Saúde da criança: Crescimento e desenvolvimento. Brasília, DF: Autor. Recuperado em http://bvsms.saude.gov. br/bvs/publicacoes/saude_crianca_crescimento_desenvolvimento.pdf
Ministério da Saúde. (2012b). Aleitamento materno, distribuição de leite e fórmulas infantis em estabelecimentos de saúde e a legislação. Brasília, DF: Autor. Recuperado em http://bvsms.saude. gov.br/bvs/publicacoes/aleitamento_materno_ distribuicao_leite.pdf

Ministério da Saúde. (2013a). Atenção à saúde do recém-nascido: Guia para os profissionais de saúde. Intervenções comuns, icterícia e infecções. Brasília, DF: Autor. Recuperado em http://bvsms.saude.gov.br/bvs/publicacoes/atencao_saude_recem_nascido_profissionais_v3.pdf

Ministério da Saúde. (2013b). PNAN: Política Nacional de Alimentação e Nutrição. Brasília, DF: Autor. Recuperado em http://bvsms.saude.gov. br/bvs/publicacoes/politica_nacional_alimentacao_nutricao.pdf

Organização Pan-Americana de Saúde. (2013). La OPS/OMS llama a apoyar a las madres que amamantan para asegurar la lactancia exclusiva hasta los 6 meses. Recuperado em http:// www.paho.org/hq/index.php?option=com_co ntent\& view $=$ article \&id=8914\%3 Apahowho-urges-support-for-nursing-mothers-to-ensure-exclusive-breastfeeding-up-to-six-months$-\&$ catid $=740 \% 3$ Anews-press-releases\&Itemid $=$ 1926\&lang $=$ es\&Itemid $=1926$

Paiva, S. S., \& Galvão, M. T. G. (2004). Sentimentos diante da não amamentação de gestantes e puérperas soropositivas para HIV. Texto Contexto Enfermagem, 13(3), 414-419. doi:10.1590/ S0104-07072004000300011

Rahalkar, A., Phalke, D. B., \& Phalke, V. D. (2014). A study of breastfeeding and complementary feeding practices with emphasis on misconceptions amongst the women with under two year children in rural area. International Journal of Medical Research \& Health Sciences, 3(4), 851855. doi:10.5958/2319-5886.2014.00013.7

Santiago, L. B. (2013). Manual de aleitamento materno. Barueri, SP: Manole.

Secretaria Estadual de Saúde de Minas Gerais. (2012). Cartilha da gestante. Mães de Minas: A vida merece esse cuidado. Recuperado em http://www.saude.mg.gov.br/images/documentos/Cartilha\%20da\%20gestante.pdf

Secretaria Estadual de Saúde do Amazonas. (2012). Plano estadual de saúde: PES- 2012-2015. Recuperado em http://www.saude.am.gov.br/ docs/pes/pes_2012-2015.pdf 
Secretaria Estadual de Saúde do Mato Grosso do Sul, Fundação Nacional de Saúde, Departamento de Saúde Indígena. (2007a). Cartilha aleitamento materno: Alimento da mãe para o filho. Etnia: Guarani/Kaiowá. Campo Grande, MS: Secretaria Estadual de Saúde do Mato Grosso do Sul.

Secretaria Estadual de Saúde do Mato Grosso do Sul, Fundação Nacional de Saúde, Departamento de Saúde Indígena. (2007b). Cartilha aleitamento materno e introdução de novos alimentos: Sua importância. Etnia: Terena. Campo Grande, MS: Secretaria Estadual de Saúde do Mato Grosso do Sul.

Secretaria Estadual de Saúde do Mato Grosso do Sul, Fundação Nacional de Saúde, Departamento de Saúde Indígena. (2007c). Cartilha aleitamento materno e introdução de novos alimentos: Sua importância. Etnia: Kadiweu. Campo Grande, MS: Secretaria Estadual de Saúde do Mato Grosso do Sul.

Secretaria Estadual de Saúde do Mato Grosso do Sul. (2007d). Amamentação em Libras. Campo Grande, MS: Autor.

Secretaria Municipal da Saúde de Curitiba - PR. (2011). Alimentação infantil: Cartilha de orientação aos pais (2. ed.). Recuperado em http:// www.crn8.org.br/uploads/arquivo/b517902a83b8b7acd6cf009cb1779dc0.pdf
Senado Federal. (1988). Constituição da República Federativa do Brasil. Brasília, DF: Autor. Recuperado em http://www.planalto.gov.br/ ccivil_03/constituicao/constituicaocompilado. $\mathrm{htm}$

Thakur, N. A., Holambe, V. M., \& Wadagale, A. V. (2015). Survival analysis of duration of exclusive breast feeding using life table and hazard function. JKIMSU, 4(1), 88-94.

World Health Organization. (2009). Infant and young child feeding: Model chapter for textbooks for medical students and allied health professionals. Retrieved from http://www. who.int/maternal_child_adolescent/documents/9789241597494/en/
Recebido: 05/02/2015

$1^{a}$ revisão: 24/08/2015 Aceite final: 02/09/2015 